\title{
DONDE TE LLEVE EL AMOR. Nuevos sujetos de estudio, nuevas condiciones de producción del conocimiento y sus [re]planteamientos etnográficos
}

\author{
Jordi ROCA GIRONA \\ Universidad Rovira i Virgili \\ jordi.roca@urv.cat
}

\begin{abstract}
WHEREVER LOVE TAKES YOU. New study subjects, new conditions of knowledge production and its ethnographic approches
\end{abstract}

Resumen: El artículo pretende reflexionar sobre algunos de los retos que la creciente movilidad y el aumento de flujos transnacionales y realidades globales, por una parte, y la producción de conocimiento a través de proyectos colectivos subvencionados, plantea a la etnografía. La aportación se basa en la experiencia de cuatro proyectos de investigación, realizados en el marco del Plan Nacional de I+D y llevados a cabo desde el año 2006 hasta la actualidad. Estos proyectos abordan un fenómeno en última instancia móvil, en gran medida virtual y múltiplemente situado como es el de las migraciones o movilidades por razones amorosas. La investigación ha considerado localizaciones dispares y ha recorrido los consiguientes itinerarios de hombres y mujeres que se buscan y se encuentran -y se relacionan por múltiples vías y deciden cambios de residencia- en el actual y casi ilimitado supermercado amoroso. Esto ha supuesto tomar el espacio virtual como una unidad de observación más y también como una herramienta más de obtención de información.

Abstract: The increasing mobility, along with the consequent increase of the transnational flows and globalized realities on the one hand, and the production of knowledge through subsidized collective projects on the other, are posing some new challenges to the ethnography. This article aims to reflect on some of these challenges, based on four research projects carried out within the framework of the National R \& D Plan between 2006 to the present, on a phenomenon ultimately mobile, virtual and multiple-sited as the migrations or mobility for love reasons. Multiple locations have been visited, and love itineraries between men and women from different origins have been reconstructed. These men and women search and find one another -relate to each other and decide to change residences- within the present and almost unlimited love supermarket. Consequently, in these projects the virtual becomes an observation unit as well as a tool to obtain information.

Palabras clave: Etnografía multi-situada. Movilidad amorosa. Parejas mixtas. Investigación en equipo. Proyectos competitivos financiados

Multi-sited ethnography. Loving mobility. Mixed couples. Team research. Subsidized competitive projects. 


\section{(Algunos) Nuevos retos etnográficos ante la era global}

Sin ánimo de adentrarme en una discusión sobre la naturaleza del método etnográfico, no me resisto a mostrar, apenas, algunas de las principales características idiosincráticas -no consensuadas del todo, seguramente- de la etnografía, en la medida que guardan una relación directa y evidente con algunas de las cuestiones que voy a plantear en las líneas siguientes. La etnografía, que es un elemento del triángulo antropológico, juntamente con la comparación y la contextualización (véase Pujadas, Comas y Roca, 2010), según la lejana definición de Conklin de 1968 "requiere un largo período de estudio íntimo y de residencia en una comunidad pequeña bien determinada, el conocimiento de la lengua hablada y la utilización de un amplio abanico de técnicas de observación, incluyendo largos contactos cara a cara con los miembros del grupo local, participación en algunas de las actividades de este grupo y un mayor énfasis en el trabajo intensivo con los informadores que en la utilización de datos documentales o de encuesta" (1988: 153)․ Más cercana en el tiempo, la definición de Hammersley y Atkinson, en su conocido manual, la presenta "simplemente (como) un método de investigación social, aunque sea de un tipo poco común puesto que trabaja con una amplia gama de fuentes de información, (en la que) el etnógrafo, o la etnógrafa, participa, abiertamente o de manera encubierta, de la vida cotidiana de personas durante un tiempo relativamente extenso, viendo lo que pasa, escuchando lo que se dice, preguntando cosas; o sea, recogiendo todo tipo de datos accesibles para poder arrojar luz sobre los temas que él o ella han elegido estudiar" (1994: 15). Como ha señalado Ferrándiz tras analizar un puñado de definiciones de diversos autores, existe una coincidencia en que la etnografía exige la presencia del investigador o investigadora en el campo estudiado (2011: 13).

En efecto, si comparamos atentamente las dos definiciones aportadas observamos cierta coincidencia en señalar como necesaria esta presencia no puntual sino consistente (en un caso se la caracteriza como larga y en el otro como relativamente extensa) de la investigadora o investigador en el campo, la cual implica participación directa en la vida de las personas y una relación cara a cara con ellas. Dicha comparación nos arroja también matices y diferencias entre ambas. Así, en tanto que la definición más clásica habla de una comunidad pequeña y de un grupo asociada a ella, derivándose implícitamente que ellos son los objetos/sujetos de estudio de la etnografía, la de Hammersley y Atkinson lo hace simple y vagamente de personas que se relacionan con los temas objeto de interés del etnógrafo o etnógrafa. De igual modo, si bien en ambos casos se alude a la utilización de un registro diverso de datos, en el primero se privilegian explícitamente las fuentes orales, en tanto que en el segundo la naturaleza de los datos se subordina en cierto modo a los objetivos de la investigación.

Los matices y diferencias entre ambas definiciones, gestadas en períodos históricos distintos, entroncan y están íntimamente relacionados, a mi entender, con el tránsito de una etnografía asociada a los estudios de comunidad (no en balde la palabra etnografía, además de hacer referencia a un tipo de metodología, que es como aquí la abordamos, posee también el significado del producto resultado de la elaboración e interpretación hecha por el investigador o investigadora del conocimiento adquirido) hacia otra que debe (sobre) vivir en la era global.

Los estudios clásicos de comunidad, en efecto, basados en el trabajo de campo, iniciados por Boas y consolidados por Malinowski, bajo la influencia del estructural-funcionalismo, constituyen un tipo de aportaciones de carácter sincrónico y atemporal en donde lo fundamental es delimitar una unidad de observación -la comunidad- que pasa a constituirse también en la unidad de análisis. El lugar de investigación es a su vez el objeto de investigación

1 Tanto en esta como en la cita siguiente, las cursivas son mías. 
(Roca, 2010). De hecho, como ya señaló Latour, buscar aquello que "más se asemeje a los ámbitos tradicionales de estudio" (1993: 149) formó parte importante de ese intento de legitimación y, de algún modo, redundó en la reproducción de la "pequeña comunidad nativa" en la propia sociedad del antropólogo, reforzando así la practica etnográfica tradicional (Perret, 2011: 6).

Así, como señaló Pío Navarro (1984: 63) a partir del análisis de veinticuatro definiciones de comunidad, podrían identificarse quince aspectos sustantivos inherentes a la misma: residencia común, continuidad temporal, relaciones sociales (inter-individuales, estructurales e institucionales), pequeñez, aislamiento, autonomía, autosuficiencia, vida social completa, homogeneidad, consenso cultural, representaciones colectivas, auto-identificación, cohesión, separación de los demás y capacidad de acción. Resulta obvio que cuando la etnografía se centra exclusivamente en una reducida unidad de observación -una comunidad- y descuida la consideración de unidad o unidades de análisis más generales en las que aquella se inserta, los estudios pueden caer fácilmente en el localismo y/o el folklorismo y carecer de capacidad explicativa. Sin embargo, por otra parte, cuando se ensaya un proceso de comunicación o contacto entre estos dos niveles señalados se advierte que éste no está a salvo de algunos peligros a considerar y que son perceptibles en dos tipos posibles de error que ha señalado Terradas (1991): el metonímico y el metafórico. El primero consiste en tomar la parte por el todo, que en este contexto significaría creer que todo lo que tiene sentido en un lugar y pasa en otros lugares, debe tener también en esos otros lugares el mismo sentido que en el primero. El error metafórico, por su parte, supone creer que hay lugares privilegiados que contienen toda la fenomenología del nivel general, es decir, que lo que ocurre en el marco de un conjunto reducido de personas es cualitativamente idéntico a lo que sucede en otros niveles a mayor escala. La pretensión, en este caso y dicho de forma muy sencilla, es la de querer embutir el mundo en una taza de café, decretando que la comunidad estudiada es el país al que pertenece en pequeño, o bien que este país es la comunidad estudiada en grande.

El interés y la necesidad de lograr una síntesis deseable que permita pasar de lo local a lo ecuménico, de lo particular a lo general, con el riesgo señalado de caer en el error metafórico, se pone en evidencia en muchas investigaciones. Frankenberg (1980: 40), por ejemplo, al repasar en su día los estudios de comunidad británicos señala:

Entre los diferentes estudios de que disponemos en este momento, este es el que se acerca más a un microcosmos del país: una Gran Bretaña en miniatura. En Banbury se encuentran representados casi todos los tipos de industria y comercio y existe toda una gama de clases sociales y grupos de estatus.

Geertz (1989: 33), por su parte, ha expresado de forma aguda una crítica al respecto, señalando, con ironía, que:

"La idea que se puede encontrar la esencia de sociedades nacionales, de civilizaciones y de grandes religiones en las llamadas pequeñas ciudades y pueblos 'típicos' es un auténtico disparate. Lo que se encuentra en las pequeñas ciudades y pueblos es ( $¡$ ay!) vida de pequeñas ciudades y pueblos. Si la importancia de los estudios localizados y microscópicos dependiera realmente de esta premisa -de que captan el mundo grande en el pequeño-, estos estudios estarían carentes de toda relevancia".

De todo ello se desprende, pues, que la clave de la cuestión es tener bien claro que el lugar de investigación (la comunidad, la unidad de observación) no es (salvo en estudios de comunidad o monografías clásicas) el objeto de estudio. El nivel local, así, se configura como una unidad que debe permitir la comprensión a través de la concreción.

A finales del siglo pasado, diversos autores todavía daban cuenta de que el trabajo de campo etnográfico permanecía exento de una revisión profunda (Comaroff, 1992), siendo 
así que podía afirmarse que el lugar, el dónde de la etnografía, se había naturalizado y pasado a formar parte del sentido común del quehacer antropológico y, por lo tanto, dejado de lado como problema metodológico (Ferguson y Gupta, 1997).

Como he desarrollado en otro lugar (Roca, 2010a), la superación, en cierto modo, de la etnografía de carácter localista y los estudios de comunidad clásicos pasó por el desarrollo, hace unas décadas, de las etnografías de orientación temática, que permitieron concretar en un nivel local, observable y por tanto aprehensible, el análisis de cuestiones y procesos de carácter general, abordando elementos que no podemos explicar sin referirnos a este contexto general pero que, al mismo tiempo, es en el marco de esta unidad microscópica de observación que podemos acceder mejor a su comprensión. La investigación, desde esta perspectiva, se basa en mantener una actitud de feedback, de dialéctica permanente entre ambos niveles. Este tipo de etnografía ha supuesto una suerte de transición hacia la llamada etnografía multi-situada (Marcus, 1995), una práctica menos marcada por el trabajo de campo, concebido al estilo clásico como la estancia de larga duración del etnógrafo/a en un solo lugar de escala reducida. Este tipo de aproximación se caracteriza por una deslocalización de la etnografía, que incorpora como material etnográfico tanto las observaciones directas de campo y las entrevistas, como el análisis de la producción literaria, el tratamiento mediático de determinadas noticias, el discurso político, los informes económicos, tecnológicos e industriales, las tendencias de la moda, los análisis sobre el consumo, la producción artística, el uso social de nuevas tecnologías, como Internet, o el análisis de los nuevos movimientos sociales emergentes, entre otros inputs informativos (Roca, 2010a: 265).

La epistemología en que se fundamenta esta nueva perspectiva metodológica tiende a situarse simultáneamente en dos polos de dicotomías del tipo local / global o vida cotidiana / sistema, superando la tendencia clásica al localismo y al particularismo. La construcción de los objetos de estudio resulta generalmente, sin embargo, más compleja, por lo que se requiere a menudo de la cooperación de especialistas de diferentes campos del conocimiento humano.

Como destaca Marcus: "La etnografía de una formación cultural dentro del sistema mundial es también una etnografía del sistema y, por tanto, no puede ser entendida sólo en términos de una puesta en escena localizada convencional, propia de una investigación etnográfica" (1995: 99; la traducción es nuestra).

Finalmente, otro rasgo característico de este enfoque es el distanciamiento respecto de la construcción de objetos de estudio subalternos, que era una tendencia muy marcada hasta hace bien poco.

En otro lugar (Roca, 2014: 306) señalábamos que la etnografía repele y se resiste a los modelos de investigación rígidos, puesto que una de sus principales bazas epistemológicas reside en el diseño de la investigación en gran medida de forma pareja a la realización de la misma. Esto no quiere decir, como se ha sugerido en ocasiones, que en la etnografía valga todo y no existan premisas ni controles de ningún tipo. Simplemente que estos no son rígidos ni se dan de forma previa, e inamovible, a la investigación. De igual modo, la "imaginación etnográfica" (Willis, 2000), surgida de un contexto en el que el/la investigador/a detenta una posición absolutamente central, debe asumir que el comportamiento y la experiencia humanas son muy variables y localmente específicos, sin perder de vista, no obstante, los distintos niveles relevantes del contexto en los que esto sucede, ya sean regionales, nacionales o globales, coloniales o poscoloniales (Ferrándiz, 2011: 14).

No debe extrañarnos pues que sean diversos los autores que se han aventurado a establecer algunos principios básicos del método etnográfico y que, en consecuencia, podamos contar con un corpus de normas y/o criterios o consejos al respecto. Entre ellos, por ejemplo, la necesaria estadía prolongada en el campo; la participación en la vida del grupo o las personas estudiados; la conjugación, por parte del etnógrafo, de los verbos observar, escuchar y preguntar; el aprendizaje de la lengua nativa; la necesidad de ahuyentar prejuicios, 
ideas previas y juicios de valor; el no forzar, intrusivamente, la generación de información; el ejercer la reciprocidad con los informantes; etc.

\section{Los nuevos retos etnográficos ante las nuevas formas hegemónicas de producción del conocimiento}

La base de mi aportación y correspondiente reflexión la constituyen una serie de cuatro proyectos de investigación, realizados sucesivamente entre 2006 y 2018 y de los que he sido Investigador Principal, sobre un mismo objeto de estudio general: el de las que he denominado migraciones por amor $^{2}$ y las consiguientes parejas, uniones o matrimonios mixtos o binacionales resultantes. Se trata, por lo demás, de proyectos financiados por distintos Ministerios del gobierno de España dentro del Plan Nacional de I+D+I ${ }^{3}$ (Véase Tabla 1), lo que supone su concurrencia previa a una convocatoria competitiva y su adecuación a una estructura estandarizada que impone ritmos y pautas específicos de realización difícilmente eludibles.

En nuestro caso nos hallábamos, pues, ante la necesidad de adecuarnos a unos proyectos altamente estandarizados con una voluntad de aplicar, en alguna medida, el método etnográfico. La solicitud debe tramitarse mediante una aplicación única para todos los proyectos, sean de la índole/disciplina que sean. Como se muestra en la Figura 1 no es posible presentar un proyecto bajo la rúbrica de "Antropología", puesto que no aparece la opción. En nuestro caso escogimos como Área temática "Ciencias Sociales", como Sub-área temática "Sociología” y como Áreas preferente y secundaria "Ciencias Sociales" e "Historia y Arte".

\begin{tabular}{|c|c|c|c|c|c|}
\hline TÍTULO PROYECTO & PERÍODO & FINANCIACIÓN & $\mathbf{N}^{\circ}$ INVESTIGADORAS/ES & $\mathbf{N}^{\circ}$ ENTREVISTAS & ESTANCIAS DE CAMPO \\
\hline 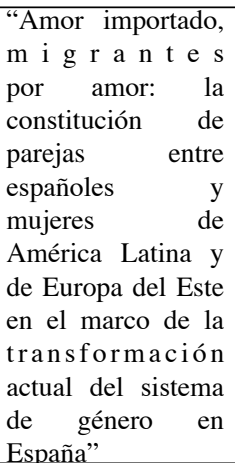 & 2006-08 & $34.127 €$ & 5 & 72 & $\begin{array}{l}\text { Kiev } \\
\text { (Ucrania); } \\
\text { Rio de Janeiro } \\
\text { (Brasil) }\end{array}$ \\
\hline
\end{tabular}

2 Algunos de los textos surgidos de estos proyectos, en donde se presentan distintos aspectos y resultados de los mismos, pueden encontrarse en Roca (2007, 2009, 2009a, 2011, 2011a, 2014, 2016, 2016a), Roca et al. (2013), Roca, Anzil y Martínez (2015), Roca y Allué (2016), Anzil, Roca e Yzusqui (2016), Djurdjevic y Roca (2016). 3 “Amor importado, migrantes por amor: la constitución de parejas entre españoles y mujeres de América Latina y de Europa del Este en el marco de la transformación actual del sistema de género en España" (Ref. 47/05, Programa Nacional de Promoción General del Conocimiento, INMU - Instituto de la Mujer, MTAS - Ministerio de Trabajo y Asuntos Sociales. 2006-2008; “Amores transnacionales: constitución y desarrollo de parejas mixtas en España” (Ref. CSO2009-10187. Proyectos de Investigación Fundamental no orientada, MCIN, Plan Nacional I+D+I.2010-2011; "Parejas mixtas residentes fuera de España: relaciones de género, dinámicas sociales y conexiones transnacionales". Plan Nacional de I+D+I, Ministerio de Economía y Competitividad (CSO201233565): 2013-2015; "Roles de género e interculturalidad de las parejas mixtas en España: una investigación cualitativa longitudinal" Plan Nacional de I+D+I, Ministerio de Economía y Competitividad (CSO2015-65531-P): 2016-2018. 


\begin{tabular}{|c|c|c|c|c|c|}
\hline 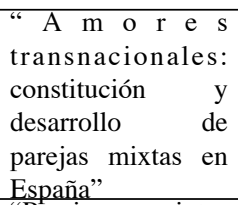 & 2010-12 & $53.000 €$ & 10 & 80 & $\begin{array}{l}\text { Natal y Sao Paulo } \\
\text { (Brasil) }\end{array}$ \\
\hline $\begin{array}{l}\text { "Parejas mixtas } \\
\text { residentes fuera de } \\
\text { España: relaciones } \\
\text { de género, } \\
\text { dinámicas sociales } \\
\text { y conexiones } \\
\text { transnacionales" }\end{array}$ & 2013-15 & $22.500 €$ & 8 & 30 & $\begin{array}{l}\text { México DF } \\
\text { (México); } \\
\text { Rio de Janeiro } \\
\text { (Brasil) }\end{array}$ \\
\hline $\begin{array}{l}\text { "Roles de género } \\
\text { e interculturalidad } \\
\text { de las parejas } \\
\text { mixtas en España: } \\
\text { una investigación } \\
\text { c u a } 1 \text { i t a t i v a } \\
\text { longitudinal" }\end{array}$ & 2016-18 & $42.100 €$ & 10 & 40 & \\
\hline
\end{tabular}

Tabla 1: Relación de Proyectos financiados por el Plan Nacional de I+D+I (Elaboración propia a partir de datos de las entidades financiadoras)

Los solicitud de un proyecto de $\mathrm{I}+\mathrm{D}+\mathrm{I}$, de acuerdo con este carácter estandarizado al que hacíamos referencia, obliga, por ejemplo, a formular hipótesis, a constituir un grupo de investigación, a elaborar un cronograma (Véanse las Figuras 2 y 3), y, en caso de ser concedido, a realizar sendos informes de seguimiento al final del primer y el segundo año de realización del proyecto, entre cuyos apartados a cumplimentar está, dentro de la "Difusión de los resultados del proyecto", las "Publicaciones científico-técnicas (con peer-review) derivadas del proyecto y patentes".

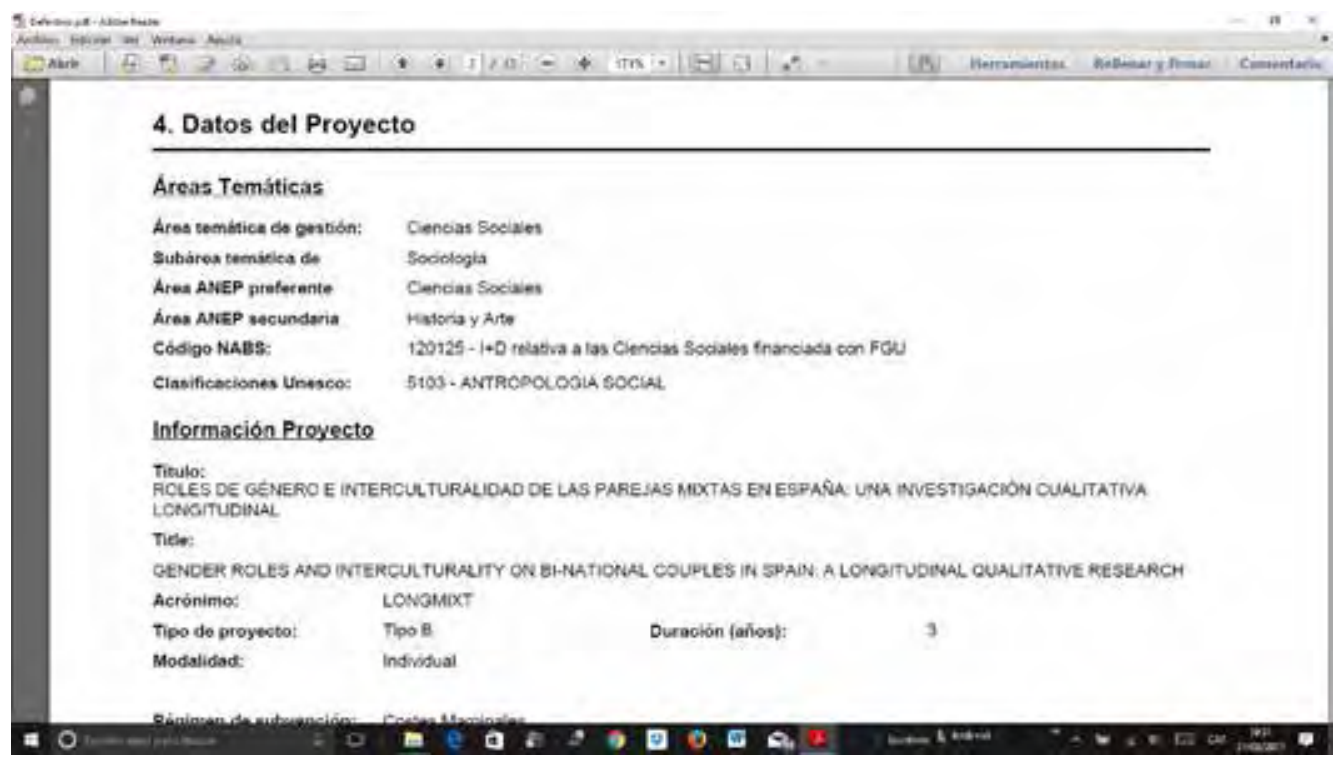

Figura 1: Formulario de solicitud de Proyectos Excelencia y Proyectos Retos del Plan Nacional de I+D+I. Captura de pantalla del Documentos Definitivo de Solicitud. 


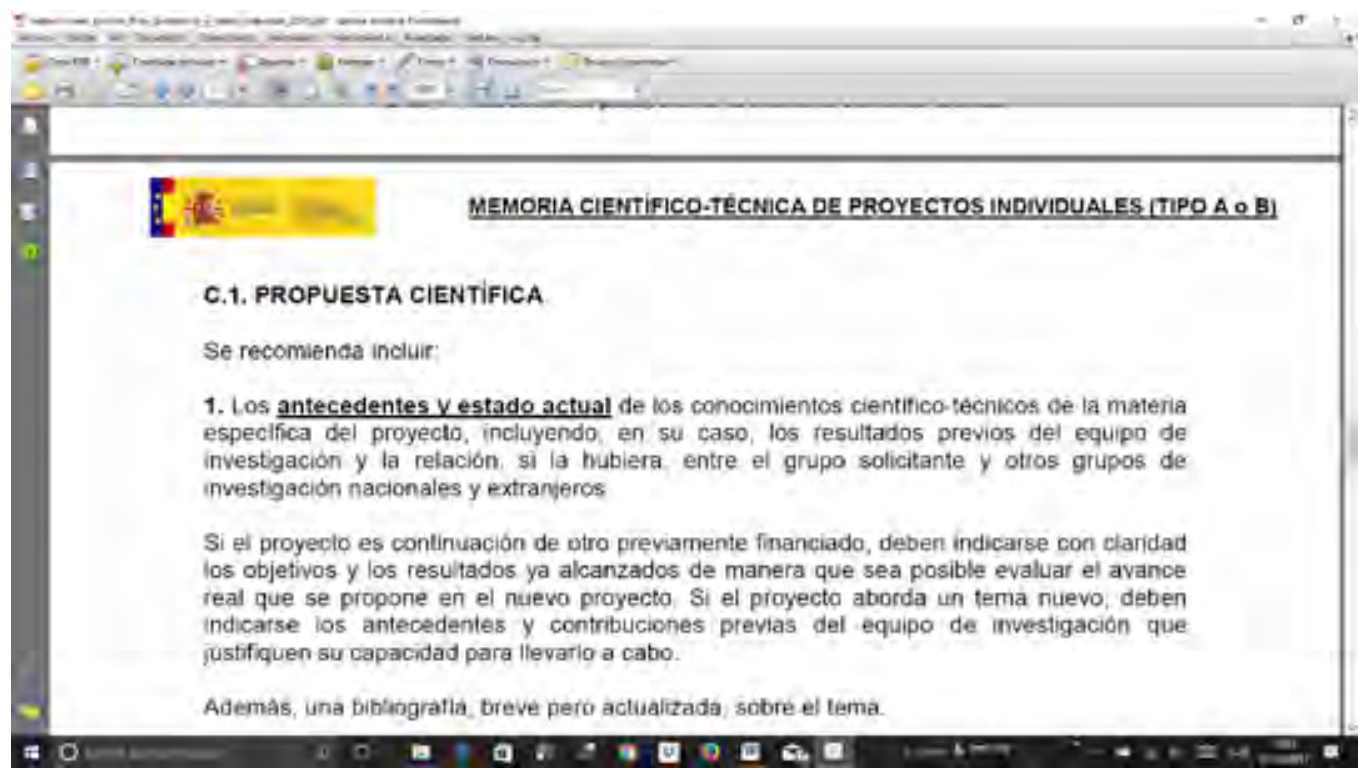

Figura 2: Formulario de la Memoria científico-técnica de Proyectos Excelencia y Proyectos Retos del Plan Nacional de I+D+I. Captura de pantalla.

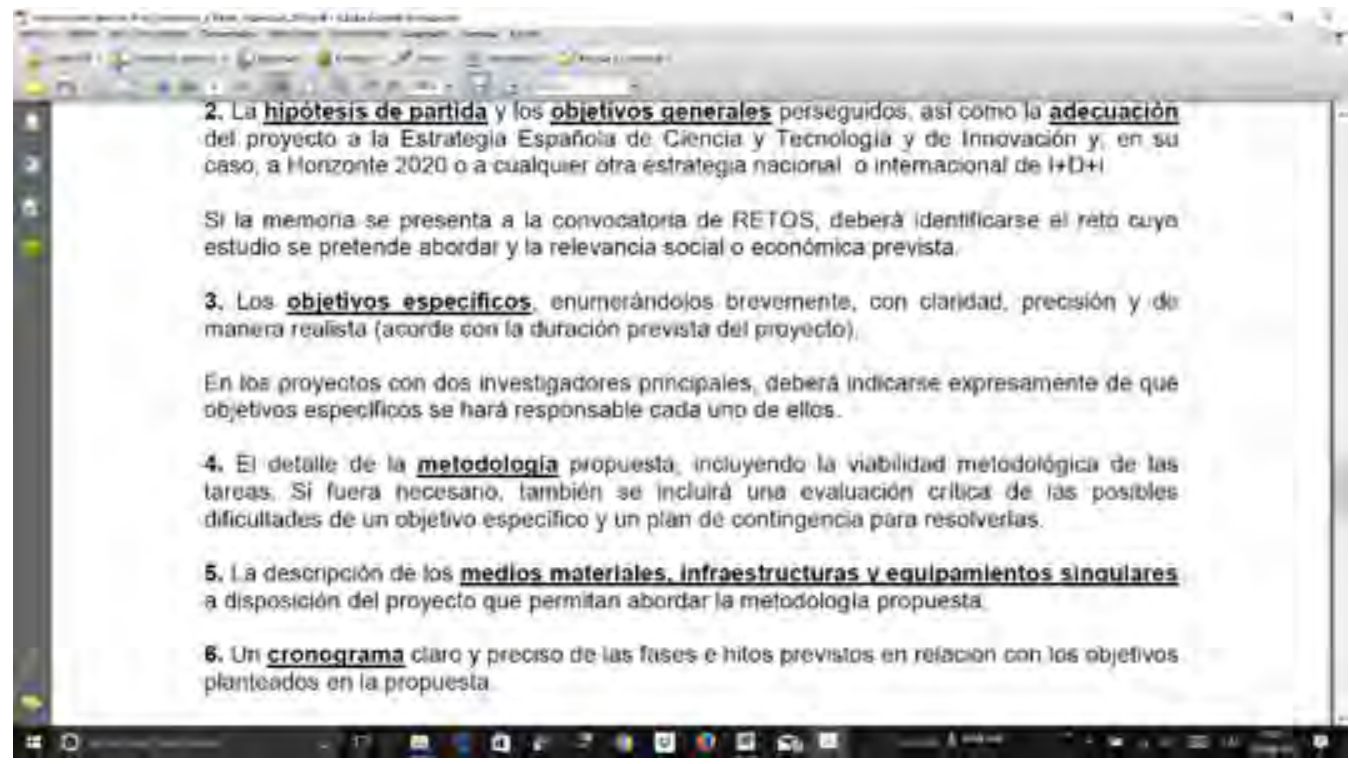

Figura 3: Formulario de la Memoria científico-técnica de Proyectos Excelencia y Proyectos Retos del Plan Nacional de I+D+I. Captura de pantalla. 
Las hipótesis, afirmaciones previas que la investigación deberá validar o falsear, no son indigestas al quehacer etnográfico en tanto que suponen una suerte de violación de aquel principio que aconsejaba eliminar las ideas previas -de hecho, quién no las tiene, etnógrafos incluidos- sino más bien porque implican organizar y estructurar la investigación de un modo que atenta, o puede atentar, contra la naturaleza misma de la aproximación etnográfica, al situar el motor de la investigación en la generación de datos encaminados de forma principal y deliberada a la falsación o verificación de las hipótesis planteadas.

La etnografía, por otra parte, se ha construido sobre la base de la gran narrativa del etnógrafo como una especie de héroe cultural solitario, enfrentado a las casi siempre duras condiciones del campo y de sus habitantes ${ }^{4}$, por lo que su práctica casa mal con la constitución de un grupo de investigación. Como decía Rivers (1975), el trabajo de campo antropológico es indivisible. Así como algunas de las técnicas más paradigmáticas de la metodología cuantitativa, como la de la encuesta, son difícilmente ejecutables sin un trabajo en equipo que conlleva una clara división intelectual del trabajo -quienes elaboran el cuestionario de encuesta no acostumbran a ser los mismos que lo distribuyen y cumplimentan ni que tabulan los datos y, en ocasiones, que los analizan-, la etnografía, que utiliza de forma privilegiada técnicas de carácter cualitativo, pero que no se agota en ellas ni significa lo mismo, se ha sustentado y sigue sustentándose en buena medida en el paradigma de la indivisibilidad entre quien recoge la información y quien la organiza, la clasifica y la analiza. Ahora bien, también es cierto que el hecho de llevar a cabo cada vez más el trabajo de campo at home sobre personas y temas ya casi imposibles de delimitar localmente y con una relativa facilidad de acceder a numerosísima información sobre ellas y ellos hace esta empresa cada vez más ardua para su abordaje en términos individuales y más proclive a la necesaria investigación no sólo en equipo sino también multidisciplinariamente.

La generación de la información en una investigación etnográfica, por lo demás, es difícilmente previsible y programable en términos de realización de un cronograma que abarque los tres años de duración prevista del proyecto y que establezca, por tanto, cuánto tiempo nos llevará la incorporación al campo, el establecimiento del rapport con el grupo o las personas objeto de estudio y la correspondiente disponibilidad de informantes o la determinación de la saturación informativa. Se dice que decía Evans-Pritchard, por ejemplo, que el tiempo estimado entre la investigación y divulgación de los resultados era de aproximadamente 10 años, en tanto que a menudo, basándose supuestamente en el trabajo de Malinowski en las Trobriand, se estipula el tiempo mínimo de estancia en el campo sobre los dos años. En este sentido, más insólita resulta aún la necesidad, dentro de la rendición de cuentas provisionales de la investigación, de consignar las publicaciones resultantes de la misma al final ya del primer año. Parece claro, obviamente, que en el caso que nos ocupa, el paradigma hegemónico de las ciencias llamadas duras, que pueden y deben planificar experimentos de laboratorio, por ejemplo, y publicar resultados provisionales de los mismos de forma casi inmediata y con una retahíla de autores por artículo, constituye el modelo de referencia para el común de las disciplinas científicas.

\section{La articulación de cuatro proyectos sobre movilidades amorosas y migraciones por amor}

Antes de entrar directamente a las cuestiones relacionadas con las técnicas y la metodología presentes en los proyectos de investigación que sirven de base a esta aportación, voy a presentar unos datos breves sobre el objeto de estudio para situar al lector. Las

\footnotetext{
4 Los ejemplos son innumerables, pero es especialmente en los diarios de campo -que desde hace un tiempo han empezado a considerarse material publicable- en que la figura del etnógrafo (re)presentando papeles diversos ha emergido con más fuerza. Véanse, por ejemplo, Malinowski (1989), Barley (1989), Rabinow (1992) o García Jorba (2000).
} 
migraciones por amor ${ }^{5}$, y la correspondiente formalización de las denominadas uniones, parejas o matrimonios mixtos o binacionales, han existido siempre, pero es sólo hasta los años 90 del pasado siglo que aparecen de manera generalizada y relevante. La búsqueda global de cónyuge, en este sentido, dibuja unas rutas que van de los "países ricos" del norte global -Occidente Europeo, Norteamérica y Asia-Pacífico- a países del denominado sur global -regiones del Caribe, Latinoamérica, el Este de Europa y el Sudeste Asiático. La literatura existente sobre uniones mixtas se integra en el creciente interés científico sobre cómo las relaciones sociales se han tornado cada vez más dispersas geográficamente, impersonales, mediatizadas por, e implicadas en, amplios procesos político-económicos, así como en la atención académica cada vez mayor a la intensificación y compleja interconectividad de los procesos locales y globales. Son muchos los trabajos que desde la segunda mitad de los años 80 se ocupan de describir y analizar las conexiones románticas entre hombres y mujeres de distintos países de estas regiones citadas (véanse, para una panorámica general que incluye referencias a contribuciones particulares de movilidad entre países concretos, entre otros a Constable, 2003, Williams, 2010, Charsley, 2012, Roca et al., 2013).

En España, en particular, contamos con datos estadísticos sobre matrimonios mixtos o binacionales desde el año 1996. En este año hubo 8.009 matrimonios mixtos, un $4.13 \%$ del total de matrimonios celebrados en España, mientras que, en 2009, que es el año en que se alcanza el porcentaje más alto de este tipo de matrimonios, se contabilizaron un total de 29.282, el 16,8 \% de todos los matrimonios celebrados en España. De los datos estadísticos de matrimonios binacionales heterosexuales celebrados en España desde 1996 hasta la actualidad se desprende que el $58 \%$ de ellos corresponden a uniones entre un hombre español y una mujer extranjera, en tanto que el $42 \%$ estarían formados por una mujer española y un hombre de una nacionalidad distinta. Las esposas extranjeras proceden de América (61 $\%)$, Europa -principalmente de la Europa oriental- (28\%), África (9\%) y Asia (2\%). Los maridos extranjeros, por su parte, son de América (38\%), Europa -principalmente de los países occidentales europeos- $(34 \%)$, África $(24 \%)$ y Asia $(4 \%)^{6}$. A pesar de que los cónyuges extranjeros de ambos sexos son predominantemente de América Central y del Sur -brasileñas y colombianas por un lado y argentinos, cubanos, dominicanos y colombianos por el otro de manera destacada-, las estadísticas muestran que las mujeres españolas se casan con los africanos -marroquíes y nigerianos especialmente- o con los europeos de los países vecinos - italianos, ingleses, franceses de preferencia- con más frecuencia de la que lo hacen los hombres españoles; y que los hombres españoles se casan más con mujeres latinoamericanas, principalmente de las nacionalidades ya señaladas, o eslavas -rusas y ucranianas en primer lugar- de lo que las mujeres españolas con hombres de esos orígenes. Esta distribución de orígenes abarca tanto países con un pasado colonial vinculado a España como otros geográficamente cercanos. Por otra parte, no es posible separar ni el aumento de las parejas mixtas ni el origen de los cónyuges extranjeros de los profundos cambios que se produjeron en el sistema español y sus relaciones de género en los años ochenta y noventa (Roca, 2011a; Roca y Urmeneta, 2013).

Estos datos nos aconsejaron centrar nuestro primer proyecto únicamente en las parejas formadas por hombres españoles y mujeres no españolas. En nuestro segundo proyecto, continuación del anterior, incorporamos la consideración privilegiada, en cambio, de las uniones entre mujeres españolas y hombres de nacionalidad no española. En ambos casos,

5 El término lo acuñé en 2006 en el marco de la presentación del primer proyecto de los cuatro sobre los que se asienta este artículo y vio la luz por primera vez en un artículo al año siguiente (Roca, 2007). Su aparición formaba parte del llamado de King (2002) a incorporar las nuevas geografías y tipologías migracionales. Si bien en más de una ocasión al inicio este término motivó cierta incomodidad e incluso rechazo entre la comunidad especializada en estudios migratorios, con posterioridad ha sido utilizada ampliamente y ha traspasado nuestras fronteras.

6 Datos obtenidos del INE (http://www.ine.es/) y elaborados por nosotros. Último acceso: 24-3-17. 
más allá de la dimensión estadística del fenómeno, que nos resultó útil para establecer la extensión del mismo y algunas de sus características, como la evolución numérica en el tiempo y el peso específico y desigual de las distintas nacionalidades, nuestro principal objetivo se centró en recoger información acerca de las motivaciones y expectativas presentes en el deseo de buscar una pareja de una nacionalidad distinta de la propia y/o de una nacionalidad específica; en las características del proceso de búsqueda y encuentro y del correspondiente período de noviazgo; y en las vicisitudes de la pareja una vez realizada formalmente la unión y viviendo ya de forma conjunta, atendiendo especialmente a aspectos de la vida doméstica tales como la distribución de tareas entre los cónyuges y el abordaje de la cuestión de los hijos, y a cuestiones pertenecientes al ámbito público, como las relaciones con las familias de orientación, el trabajo del cónyuge extranjero o la sociabilidad y las relaciones íntimas de la pareja. Todo ello desde una decidida y deliberada perspectiva de género.

En el año 2009 se alcanzó, como hemos señalado, el número máximo de matrimonios mixtos celebrados en un año en España. Desde entonces se inició un descenso ligero pero continuado de los mismos. Al igual que la aparición y crecimiento de las parejas mixtas en España se correlacionó, entre otros factores, con el aumento de la población inmigrante, su descenso se ha explicado también por la pérdida de población inmigrante en España como consecuencia de la crisis económica iniciada a finales de la década pasada. En este contexto, una parte importante de población inmigrada decidió regresar a sus países. También una parte de la población española empezó a emigrar a otros países y volvió a situar a España en la posición de país emisor de migración que había abandonado momentáneamente en las dos últimas décadas. Entre estas personas que decidieron salir de España se encontraban, obviamente, muchas parejas mixtas que, en muchos casos, decidieron empezar de nuevo en el país de origen del miembro extranjero de la misma. No en vano, Brasil ha sido uno de los lugares de destino de buena parte de la reciente emigración española y uno de los principales países de origen de las mujeres que más se casan con hombres españoles. En medio de esta coyuntura, resultaba de mucho interés llevar a cabo un proyecto con la finalidad de averiguar si las expectativas, las relaciones de género y las dinámicas sociales de estas parejas se habían transformado con el cambio de país de residencia, así como profundizar en los motivos y las razones presentes en la decisión de establecerse fuera de España, ya fuese de forma temporal o definitivamente. Lo que nos interesaba, en suma, era ver cómo el cambio en una de las variables señaladas, en este caso la localización física -un país distinto, con estructuras de género distintas- intervenía, si lo hacía, en los procesos de definición del género y de negociación de las relaciones de género en la pareja.

Estos proyectos nos aportaron una imagen estática, una "fotografía", de las cuestiones abordadas en un momento en que la mayoría de estas parejas eran de reciente constitución y se hallaban por tanto en la fase inicial de la relación. Muchas de ellas no tenían hijos, los casos de divorcio eran escasos, el cónyuge extranjero estaba aún en proceso de conseguir la nacionalidad, la convalidación de su título universitario o un primer trabajo. La mayoría de estas parejas estaban apenas ensayando fórmulas de articulación con sus respectivas familias de orientación, y algunas cuestiones que con el tiempo resultaron capitales ni siquiera se habían planteado. En este contexto, con más de 150 entrevistas realizadas y más de 190 informantes involucrados en ellas, pensé que este bagaje acumulando a lo largo de los proyectos anteriores constituía una base poderosa para abordar un nuevo proyecto, concedido e iniciado en 2016 y vigente mientras escribo este artículo, con el objetivo de superar la limitación temporal de los resultados anteriores y documentar los cambios y continuidades que han experimentado estas parejas en los años transcurridos desde que las entrevistamos, hace diez años en su caso máximo y tres en el mínimo, hasta el presente. Para ello hemos conectado con las trayectorias biográficas de una muestra del gran número de informantes a los que hemos entrevistado a lo largo de los proyectos anteriores, y nos hemos servido de la metodología de la investigación cualitativa longitudinal (ICL). 
La principal aportación de la ICL es precisamente ayudar a investigar los procesos de cambio y reproducción a lo largo del tiempo, especialmente en relación a procesos de evolución, transformación o creación de identidades. Y son precisamente los roles vinculados a la identidad de género y la negociación de la identidad cultural de la pareja como tal y de la correspondiente familia mixta a la que puede dar lugar, las dos principales unidades de análisis que, en línea con la investigación llevada a cabo en los anteriores proyectos, pretendemos abordar mediante la ICL, de cuyo diseño de investigación forman parte ineludible el tiempo y el énfasis en el cambio. Como señalan Caïs, Folguera y Formoso (2014: 14-16) es solamente a través de la incorporación de la dimensión temporal que podremos observar de forma más precisa las vinculaciones e interconexiones entre lo personal y lo social, entre el agente y la estructura, entre lo micro y lo macro y constatar su transformación a lo largo del tiempo. De este modo estaremos preparados también para profundizar en la naturaleza del cambio social, en los mecanismos y estrategias utilizados por los individuos para generar y manipular el cambio en sus vidas y ver la manera cómo el cambio estructural impacta en la vida de los individuos.

Creemos, como hemos señalado en diversas ocasiones (Roca, 2007), que las unidades de análisis pertinentes para la correcta contextualización de este fenómeno pasan por la consideración de la creciente existencia de flujos transnacionales y realidades de carácter globalizado, por la emergencia y extensión de las nuevas tecnologías de la información y la comunicación (TIC) y por el paso de una sociedad centrada en la producción a otra centrada en el consumo y en la elección. Esto nos sitúa en un escenario en donde los límites y las fronteras entre migración y movilidad se difuminan al tiempo que crece su intensidad en relación a recursos de toda índole: personas, capitales, mercancías (Featherstone, 1990; Robertson, 1992; Hannerz, 1998; Appadurai, 2001). Debe considerarse además la incidencia de estas nuevas realidades contextuales en el territorio amoroso, en el marco de la transición, con sus correspondientes mudanzas y continuidades, del amor romántico hacia nuevas fórmulas como la del amor confluente (Giddens, 2000), basado en la reflexividad -las relaciones continúan porque así lo eligen ambos miembros de la pareja-, o la de la entronización de la aventura amorosa como paradigma de la condición postmoderna (Illouz, 1998), con la correspondiente pérdida de la inocencia del amor y la afirmación de que somos turistas de nuestro propio territorio privado, en el marco del reino del individualismo que nos otorga una creciente capacidad para escoger cuándo, dónde y con quién tener relaciones sexuales y en el que el olvido es más importante que el recuerdo (Bawin-Legros, 2004).

En suma, como han documentado diversos autores (Beck y Beck-Gernsheim, 1998; Shumway, 2003; Zelizer, 2005), muchos aspectos de la intimidad y de las relaciones personales han ido comercializándose cada vez más explícitamente vinculados a los procesos globales de mercantilización o bajo la presión de las "biografías de mercado" o vidas conformadas por las demandas del mercado.

\section{El (osado) intento de llevar a cabo una investigación (presuntamente) etnográfica con unidades de observación múltiples y volátiles en el marco de un programa nacional de investigación}

Las características particulares del objeto de estudio que acabamos de presentar suscitaron una serie de implicaciones de índole metodológica. Así, el estudio de realidades vinculadas a procesos de movilidad de personas supuso la consideración de una multiplicidad de unidades de observación. El abordaje de cada unidad se hizo de forma diferencial, atendiendo a los objetivos de cada uno de los proyectos, a los recursos disponibles y a las características diferenciales de cada una de ellas. Así, no debe olvidarse que la investigación se centraba en parejas mixtas formadas por un cónyuge español y que la realización 
de la misma la llevaba a cabo un equipo básicamente español ${ }^{7}$, financiado por el gobierno español.

\section{Unidades de observación y estancias de campo.}

En nuestro caso, para los dos primeros proyectos establecimos las siguientes unidades de observación principales: las correspondientes a los países de origen de ambos cónyuges -en donde España sería la "unidad independiente" y los correspondientes países de los cónyuges extranjeros las "unidades dependientes"- y el entorno virtual, como una de las vía privilegiadas de búsqueda, encuentro y conocimiento de estas parejas mixtas que suponen la existencia de una migración amorosa por parte de uno de los cónyuges 8 . De la primera realizamos la mayor parte del trabajo de campo en España, aunque pudimos llevar a cabo también tres pequeñas incursiones en países relevantes de procedencia de esposas transnacionales de españoles: dos en Brasil y una en Ucrania.

En Brasil aprovechamos parte de los fondos del proyecto y fundamentalmente la cobertura de otro proyecto concomitante ${ }^{9}$ que nos permitió becar a una investigadora del equipo para realizar una estancia de tres meses, del 1 de setiembre al 30 de noviembre de 2011, en el marco de la cual realizó una serie de entrevistas a parejas heterosexuales formadas por una persona de nacionalidad brasileña y otra de española así como observación participante entre asociaciones de la comunidad española y catalana en Sao Paulo (Casa España don Felipe de Sorocaba; Catalonia Grup de catalans en Sao Paulo; Sociedad Hispano Brasilera de Ipiranga en Sao Paulo; Consejo de residentes españoles en Sao Paulo, etc.). Con fondos de ambos proyectos pudo costearse también la realización de trabajo de campo del investigador principal del proyecto y otra investigadora del mismo en la ciudad de Natal, al nordeste de Brasil, por espacio de tres semanas en el verano de 2011. En el curso de esta estancia se llevaron a cabo entrevistas con parejas de la misma tipología que las citadas, a agencias matrimoniales de la ciudad y entre hombres españoles con negocios en Natal y relaciones con mujeres brasileñas de la zona, así como observación participante en enclaves turísticos, como la playa de Ponta Negra y de Pipa y complejos de ocio nocturno en la ciudad de Natal.

La estancia en Ucrania se fraguó a partir del contacto con diversas agencias matrimoniales especializadas que, inicialmente, se mostraron reticentes a colaborar en la investigación. Las principales razones argüidas tenían que ver con el hecho de manifestar estar hartos de periodistas (sic) -lo que nosotros entendimos como una suerte de manifestación latente de su conciencia sobre los prejuicios hacia las parejas mixtas y especialmente hacia las agencias que las promueven- o porque solicitaban dinero a cambio de su colaboración ${ }^{10}$. Hubo

\footnotetext{
7 Excepto en el primer proyecto, en cada uno de los restantes ha habido en el equipo dos o tres investigadoras, no siempre las mismas, de universidades y centros de investigación no españoles. Concretamente tres de centros portugueses y una perteneciente a dos universidades suizas.

8 Los otros medios de búsqueda y configuración de parejas mixtas que hemos documentado serían los viajes -en el marco de un turismo de ocio, sentimental, sexual, etc.- y el país de destino fruto de un proceso migratorio previo por razones no amorosas.

9 "Migración, mercados del sexo y del matrimonio y trata de personas en los flujos de circulación entre Brasil y España". SGUM - Secretaría General de Universidades - Ministerio de Educación PHB-2010-0020-PC CINT Cooperación internacional, con Adriana Piscitelli y Jordi Roca como investigadores principales.

10 La conveniencia o no de remunerar por parte del investigador la colaboración de informantes en una investigación ha sido tratada escasamente en la literatura etnográfica, si bien los argumentos de las posiciones a favor y en contra parecen claramente establecidos y tienen que ver con el hecho de afirmar que el dinero puede corromper el vínculo entre investigadores e investigados, pudiendo llegar a provocar la invención de historias falsas con el único objetivo de percibir un dinero o bien, por el contrario, que simplemente debe tratarse como una forma de "recompensa" recíproca por el tiempo concedido por los informantes, algo que la mayoría de etnógrafos suscribe pero no en términos monetarios. En nuestro caso hemos de decir que no llegamos a remunerar monetariamente a ningún informante-colaborador, entre otras razones porque la entidad financiadora de los proyectos no contempla tal posibilidad. De todos modos, el hecho de que algunos avispados responsables
} 
dos agencias que se mostraron dispuestas a recibirnos y a contarnos las características del negocio. Con una de ellas, denominada Interdating (http://www.interdating.es/), amigasrusas. com (http://amigasrusas.com) o chicas del este (http://www.chicasdeleste.com/), puesto que aparece, aunque en apariencia no es la misma, con estos tres nombres en la red, llegamos a un acuerdo para realizar observación participante en un viaje a Kiev, pagando el precio de cualquier usuario. Éste oscilaba entre los 1.300 euros para un viaje de tres días y los 1.900 para uno de 10 días en la modalidad más económica (de alto nivel) y entre los 2.100 y los 2.500 para la versión VIP. El precio daba derecho al vuelo de ida y vuelta desde Barcelona, aunque el cliente podía contratarlo por su cuenta, la recepción y traslados al aeropuerto, el alojamiento en un apartamento céntrico de la ciudad, la asistencia durante la estancia y en la oficina de Kiev, el contacto y presentación de las chicas previamente seleccionadas o seleccionadas en la misma oficina durante la estancia y el servicio de traducción si era necesario. La empresa había iniciado su andadura en los años 90 en EE.UU. y Alemania. En 2006 se había establecido en España, donde decía tener 1000 hombres españoles afiliados. Contaba con 1020 mujeres ucranianas afiliadas. Los perfiles de los primeros no eran públicos, los de las segundas sí, aunque no tenían por qué ser necesariamente reales ${ }^{11}$.

El trabajo de campo en Kiev lo realicé del 16 al 26 de junio de 2007. Pudimos saber que durante el mes en el que realizamos nuestra estancia la agencia asistió a 39 personas procedentes de distintos países, entre ellos 6 españoles. En esta breve estancia se llevó a cabo observación diaria en la agencia y se realizaron un total de 15 entrevistas a mujeres ucranianas inscritas en la agencia y observación participante, con algunas entrevistas informales, con cuatro hombres españoles clientes de la misma, así como entrevistas a agencias matrimoniales sitas en la ciudad de Kiev y a una pareja de español y ucraniana residente en Kiev ${ }^{12}$.

En cuanto al entorno virtual, nuestro trabajo de campo consistió en la búsqueda y análisis de páginas de agencias matrimoniales de búsqueda de pareja transnacional a través de viajes e Internet (especialmente las que ofertaban relaciones con mujeres de Rusia y Ucrania y con "mujeres latinas", según su propia tipología) y en la observación en algunos foros especializados de parejas mixtas, de donde surgieron algunos futuros informantes. El rol del investigador, en primera instancia, fue el de observador no participante, y más adelante, en algunos casos, de observador participante, exponiendo su condición de investigador, las líneas generales del proyecto y solicitando la colaboración de los internautas que lo desearan. La presencia manifiesta del observador provocó, en una ocasión, un debate en el foro en cuestión acerca de su legitimidad, con posiciones polarizadas en torno al hecho de si es que se consideraba a los miembros del foro como "conejitos de indias" -posición minoritariao se entendía tal atención como una oportunidad para dar a conocer de una forma menos estereotipada, tópica y prejuiciosa su realidad.

Para el tercer proyecto las unidades de observación fueron las mismas, excepto la unidad de observación correspondiente a España, puesto que los sujetos de estudio fueron parejas heterosexuales de nacionales españoles con personas de otras nacionalidades que vivieran fuera de España. Cuando los fondos propios del proyecto financiado resultaban más necesa-

de agencia nos pidieran dinero se debe, creemos, a un error nuestro de partida que, para dar garantías de seriedad a nuestros interlocutores, a menudo al presentarnos declarábamos, para el caso del primer proyecto, que formábamos parte de un "proyecto financiado" por el Instituto de la Mujer, dentro del Plan Nacional de I+D. Uno de los responsables de agencia que accedió a colaborar con nosotros sin solicitar contrapartida económica alguna a cambio -aunque ya tuvo su "recompensa" con la contratación por nuestra parte de un paquete de viaje a Kiev para realizar observación participante en su agencia- incluyó posteriormente en la página de inicio de su portal de Internet un logo de la institución que nos financió el proyecto -el Instituto de la Mujer-, con una leyenda debajo en donde se leía: "agencia colaboradora del Instituto de la Mujer". Puede imaginarse el lector el asombro de las responsables de dicho instituto y de quien esto escribe al enterarnos de tal extremo.

11 En este ámbito es habitual la existencia del fenómeno de las llamadas "scammers", o falsos perfiles, y del "hit and run", o venta de direcciones falsas (Roca, 2011).

12 Para una exposición más extensa y detallada de este trabajo de campo puede consultarse Roca, 2011 y 2016. 
rios para poder llevar a cabo trabajo de campo en el extranjero fue precisamente cuando se nos financió con la cantidad más baja del total de los cuatro proyectos obtenidos. Aun así, conseguimos realizar trabajo de campo en el extranjero, en este caso en Rio de Janeiro y en México DF. En el primer caso, a través del proyecto, una investigadora consiguió una Beca de la Fundación Carolina de Movilidad de Profesores e Investigadores Brasil-España, que le permitió realizar trabajo de campo en Rio de Janeiro durante un mes, del 26 de enero al 28 de febrero de 2013. En el curso de esta estancia realizó seis entrevistas en profundidad a mujeres y hombres españoles que optaron por emigrar a Brasil tras iniciar (por distintas vías) una relación sentimental con un hombre o mujer brasileña, y contactó con diversas asociaciones españolas en la ciudad: la Casa de Espanha en Río de Janeiro; AJED-RJ Jóvenes Españoles; Consejo de Residentes Españoles (CRE / RJ) o la Casa de España Don Felipe II. En el caso de México DF, como investigador principal del proyecto, pude aprovechar distintas estancias de carácter académico propiciadas por invitaciones del Instituto de Investigaciones Antropológicas de la UNAM y de la Universidad Iberoamericana para llevar a cabo diversas entrevistas (concretamente seis) a parejas heterosexuales de mexicanos/ as y españolas/es residentes en la ciudad. Las entrevistas se concertaron mediante diversos anuncios en foros de páginas del estilo de "españoles por el mundo" y a través de contactos proporcionados por colegas de las citadas universidades. Estas estancias se realizaron del 7 de setiembre al 7 de octubre de 2013 y del 20 de enero al 2 de febrero de 2014.

Como puede verse, en cierto modo nos planteamos una investigación que bien podía ubicarse, en principio, bajo las etiquetas de la etnografía multi-situada o multi-local, dado que nuestro objeto de estudio era, en última instancia, móvil y múltiplemente situado, que uno de nuestros objetivos era el de seguir empíricamente el hilo conductor de procesos culturales (Marcus, 1995), y que nuestros sujetos de estudio formaban parte de procesos transnacionales y de intrínseca movilidad, tanto física como virtual; y de la etnografía virtual (Hine, 2004). Nuestro trabajo, en cierto modo, describe una comunidad global de mujeres y hombres envueltos en relaciones de noviazgo y de pareja/matrimonio y plantea la cuestión, entre otras, de cómo el desarrollo de Internet y de las distintas formas de comunicación electrónica han permitido la emergencia de nuevos tipos de comunidades imaginadas (Anderson, 1983; Appadurai, 2001) y cómo los antropólogos pueden acometer el estudio de éstas ${ }^{13}$.

\section{Incorporación de "nuevas" técnicas de obtención de información.}

La pluralidad diferenciada de unidades de observación, la idiosincrasia de cada uno de los proyectos y los recursos disponibles implicaron el uso de una variedad de técnicas. En este sentido se llevaron a cabo distintos tipos de entrevistas. Por lo que a su composición se refiere debemos mencionar las siguientes: individuales -a un solo miembro de la pareja, bien porque el otro no quería o no podía-; conjuntas -a ambos miembros de la pareja al mismo tiempo-; dobles -a los dos miembros de la pareja por separado-; y en grupo -a más de dos personas a la vez, generalmente con un mínimo de 7 y un máximo de 13, que compartían una vinculación similar con el tema central de discusión. En cuanto al formato, se utilizaron igualmente diversos medios para entrevistar, como por ejemplo cara a cara y por correo electrónico, normalmente después de conocer y contactar a un futuro informante en el ámbito virtual -chats, foros, etc.-. También por teléfono, en el caso por ejemplo de una directora de una agencia matrimonial especializada en uniones hispano-rusas situada en Asturias o de una mujer española casada con un ciudadano de la República Centroafricana que regularmente venía al País Vasco para ver a su madre y familiares y aprovechaba para escolarizar un tiempo a su hijo en el sur de Francia, y en algún otro caso de personas que se

13 Empezamos a contar ya con un buen número de trabajos que muestran, entre otras cosas, que estas comunidades son complejas, organizadas y dignas de estudio. Sólo a título de ejemplo, pueden verse Rheingold, 1996; Jacobson, 1996, 1999; Miller y Slater, 2000; Mayans, 2002; Hine, 2004. 
encontraban en ciudades españolas alejadas de Cataluña, que es donde se ubica el equipo de investigación y donde se realizaron la mayor parte de entrevistas cara a cara con miembros de parejas mixtas. E igualmente por Skype, especialmente en el tercero de los proyectos llevados a cabo, centrado en parejas mixtas con un cónyuge español residentes fuera de España, y en el cuarto, en donde parte de la muestra de parejas escogidas para revisitar bien ya no viven en España bien forman parte del grupo de parejas entrevistadas en el tercer proyecto.

En relación a esta última fórmula vale decir que más allá de permitir acceder a informantes residentes en lugares alejados a los que no puede desplazarse la investigadora o el investigador, la entrevista por Skype posee algunas potencialidades nada desdeñables. Por ejemplo, una grabación sonora de muy alta calidad y una gestión de la intimidad de la persona entrevistada controlada en todo momento por ella misma, permitiendo una relación y situación de entrevista no intrusiva ni invasiva -dando por sentado que la "aparición" de un/a antropólogo/a en la vida de una persona "convertida" en informante siempre posee algún grado de "intrusión" o "situación forzada"- puesto que la persona entrevistada escoge lo que desea mostrar y el plano cómo lo desea mostrar.

El recurso a los instrumentos y aplicaciones que permite Internet no se ha limitado al uso de Skype para la realización de entrevistas con informantes lejanos. Con algunas y algunos informantes hemos mantenido, y seguimos manteniendo, relación a través de Facebook y Messenger, WhatsApp o e-mail. Ello nos ha facilitado por ejemplo que hayamos podido acometer el actual proyecto de carácter longitudinal con mayores garantías, puesto que con una parte de nuestras/os informantes se había seguido manteniendo el contacto o, cuando menos, la posibilidad del mismo estaba latente. Estas aplicaciones nos han servido también en más de una ocasión para, tras la realización de la entrevista o de su transcripción, contactar de nuevo con un/a informante para pedir la aclaración de algún extremo o realizar preguntas de carácter puntual que se habían olvidado o que han surgido con la escucha de la entrevista.

Si bien el número de entrevistas no se fijó con antelación, como corresponde a la orientación metodológica que se siguió y al uso del criterio de la saturación informativa como pauta para decidir el final de la fase de entrevistas, sí que se realizó, con carácter orientativo y flexible, una "muestra" relevante y representativa de informantes que tuviera en cuenta las principales nacionalidades de cónyuges extranjeros de uniones binacionales con españoles/ as así como las variables interseccionales de la clase social, la edad, el estado civil previo y el nivel educativo entre otras. Además de este grupo mayoritario de informantes, compuesto por miembros de parejas mixtas, se realizaron encuentros y entrevistas con responsables de agencias matrimoniales, abogados, miembros de asociaciones de migrantes, etc. En total, a día de hoy, contamos con cerca de 200 entrevistas realizadas, transcritas, clasificadas, codificadas y, en gran medida, analizadas.

\section{Algunas cuestiones finales: ¿es posible la investigación etnográfica hoy?}

Una de las cuestiones principales que se ha querido plantear en el texto, que tiene que ver con las condiciones actuales hegemónicas de producción del conocimiento, es si se puede -y si se puede, cómo, hasta dónde- conjugar la etnografía con los proyectos de investigación obtenidos por vía competitiva y financiados por los programas de investigación estandarizados de las administraciones u organismos tanto públicos como privados. Si, por otro lado, tenemos en cuenta además las características de la sociedad actual, con un escenario configurado, entre otros, por la globalización y el post-colonialismo, bien podemos preguntarnos también si el clásico "estar allí" del credo etnográfico no se ha vuelto y ha implicado cada vez más un "estar allí, allá y aquî", cuando no un "estar por ahí". Desde luego siguen existiendo las esquinas de Foote Whyte (1971), pero ya no sólo están en la calle sino también en la red. 
El ejemplo que hemos traído a colación sugiere que podamos plantearnos preguntas como: ¿Es esto etnografía? ¿A qué podemos llamar etnografía hoy? ¿Qué es (im) prescindible del viejo paradigma etnográfico?

Tal vez, formalmente, las dos características más "antipáticas" de los proyectos que acabo de reseñar, si las enfrentamos al método etnográfico, son su desarrollo en base a un trabajo en equipo y la discontinuidad y relativa brevedad del trabajo en el campo. Como de alguna forma ha podido entreverse, la existencia de un equipo más o menos amplio de investigadoras/es permite la posibilidad -nada desdeñable en el marco de las condiciones de la vida académica- de sumar distintas estancias de campo a cargo de diferentes investigadoras, así como de disponer de un equipo, y por tanto de una perspectiva, interdisciplinario. El mayor "peligro", en mi opinión, del trabajo en equipo de una investigación de carácter etnográfico es que dé pie a una división técnica del trabajo, al estilo de lo que sucede a menudo en proyectos de carácter cuantitativo y/o experimental, en donde las investigadoras/ es que recogen la información no son los/as mismos/as que quienes la clasifican/codifican ni que las que la analizan. Si, por el contrario, la existencia de un equipo de investigación supone la participación plena, aunque con dedicaciones diferenciales por la misma lógica apuntada de las características de la vida académica, de todas/os las/os investigadoras/es en todo el proceso y en la toma de decisiones, el proyecto puede enriquecerse notablemente en algunos aspectos, aunque desde luego padecerá de otras limitaciones por otros lados.

No sé si las investigaciones que sirven de base a este texto son etnografía polifónica, multi-situada, participativa, radical, virtual, en casa, de fin de semana, inmoral... ni si hemos hecho etnografía a secas o, únicamente, trabajo de campo, que siendo un ingrediente fundamental de la etnografía no debe confundirse con ella. Lo que es seguro es que hemos hecho lo que hemos podido y sabido, lo cual por otra parte resulta ser lo habitual en la investigación con metodología no estandarizada. Cosa diferente es que luego uno cuenta -o no- lo que su sentido de la ética, del pudor, del ridículo o de la autocrítica le permiten. En cualquier caso, lo que sí resulta cierto es que tales ejemplos no constituyen, ciertamente, un paradigma de una etnografía clásica. Desde este punto de vista, pues, lo presentado no es, ni de lejos, una etnografía. Pero, por otro lado, si eso es así, podemos preguntarnos igualmente si es que es imposible abordar el estudio de un fenómeno como el de las migraciones amorosas mediante el método etnográfico. Si esto fuera así deberíamos plantearnos, como hacen Marcus y Fischer (1986) y Appadurai (1996), que la etnografía se enfrenta al desafío que supone una herramienta metodológica que muestra su posible no adecuación frente al contexto sociocultural contemporáneo. Es decir, entre los problemas que se originan en un mundo móvil, cambiante y globalizado y una herramienta metodológica como el trabajo de campo especialmente construida para el análisis de sociedades a pequeña escala y espacialmente circunscriptas a un territorio específico (Perret, 2011: 58).

La etnografía multi-situada sobre la que hemos debatido al inicio y a la que de alguna manera hemos apelado consiste, precisamente, en una práctica etnográfica deslocalizada, que se basa en la utilización de una multiplicidad de unidades de observación y participación. Busca, pues, un acercamiento a la complejidad de la realidad contemporánea por vías diferentes a las del "trabajo de campo" clásico, considerando una multiplicidad de dimensiones diferentes, tanto en lo referente al comportamiento de diferentes tipos de actores sociales, como la producción escrita o audiovisual de algunos de estos agentes sociales. Este tipo de práctica etnográfica propugna tanto el trabajo de equipo como el trabajo multidisciplinar. Indudablemente, la etnografía multi-situada, dentro de su pluralidad y heterogeneidad, es la salida que los antropólogos actuales están buscando para escapar del encorsetamiento de una práctica metodológica pensada para el estudio de sociedades exóticas y estudios de comunidad de pequeña escala, pero en cierto modo poco adecuada para el estudio y representación de las realidades contemporáneas, donde cada vez es más innegable el impacto sobre las sociedades locales de fenómenos globales. Si la epistemología clásica de la an- 
tropología enfatizaba conceptos como función, estructura y, muy secundariamente, cambio social y crisis, los nuevos modelos etnográficos buscan, por ejemplo, procesos, cadenas (de comunicación y de emigración), flujos (demográficos, económicos, culturales), conexiones o conjunciones y yuxtaposiciones entre localizaciones (Roca, 2010a: 265-266).

La respuesta, o parte de ella, a lo que venimos planteando tal vez está en que en las actuales circunstancias de producción del conocimiento y de desarrollo de la sociedad de la información y de la comunicación, el quehacer etnográfico debe re-definir alguno de sus paradigmas clásicos. Esto no debería resultar, por cierto, especialmente problemático, porque está en la esencia misma del método etnográfico ir construyendo la investigación de acuerdo con las circunstancias, imprevistas y cambiantes, que el etnógrafo va encontrándose en el campo y, por qué no decirlo también, fuera de él (la Academia, por ejemplo), y porque, como dice Geertz al hilo del "presente estado de las cosas", que define como "a la vez caótico e imaginativo, azaroso y variopinto", "ya ha sido así en otras ocasiones y pudo (la etnografía) hallarse un camino" (1989a: 158).

Así las cosas, si nos centramos en lo realmente idiosincrático y fundamental de la etnografía, su naturaleza epistemológica, no cabe duda que el contacto directo, participante, continuado, con los actores sociales para compartir sus vivencias e incorporar su perspectiva al análisis, el "estar con la gente" (de las múltiples y variadas formas que sea y cómo sea), sigue siendo la condición fundamental e indispensable para alcanzar un tipo de conocimiento relevante sobre ella. Y, vale decir, hoy no sólo puede seguir siendo posible esto, sino que incluso, en algún modo y en según qué circunstancias, es más viable aún si consideramos las potencialidades que nos ofrecen las nuevas tecnologías de la comunicación. Y de esto, a mi entender, es de lo que trata la etnografía. Después redefinamos las viejas unidades de observación y análisis que consideremos oportunas (los estudios de comunidad, de caso, etc.) y el propio ejercicio del oficio (trabajo de campo, trabajo en equipo, cierto grado de "programación de la investigación", etc.), y añadamos lo que la propia dinámica social nos demanda añadir (escenarios virtuales, móviles, internet, etc.). Quizás este ejercicio no elimine las ansiedades metodológicas de las que hablaba Marcus (1995) a propósito del desplazamiento teórico hacia la etnografía multi-local (a saber: preocupación por los límites de la etnografía, por la reducción del poder del trabajo de campo y por la pérdida de lo subalterno), pero sí que puede constituir un camino para, al menos, matizarlas y gestionarlas mejor.

Si con todo ello (o a pesar de todo ello) seguimos siendo sensibles a escuchar a la gente y a estar con ella -ya no a ponernos en la piel de ella sino a ponernos de acuerdo con ella, en la cosa-, no cabe duda de que lo que hagamos será formalmente muy distinto de lo que hacían nuestros clásicos, pero será en esencia, epistemológicamente, lo mismo. Le llamemos etnografía o cualquier otra cosa que queramos llamarle.

\section{Bibliografía}

Anderson, B. (1983). Imagined communities. Reflections on the origin and spread of nationalism. New York: Verso Press.

Anzil, V.; Roca, J. y Yzusqui, R. (2016). Amores en el registro. Mecanismos institucionales de gestión del 'amor verdadero' en los matrimonios binacionales, Scripta Nova. Revista electrónica de Geografía y Ciencias Sociales, XX (538): 1-31.

Appadurai, A. (2001). La modernidad desbordada. Buenos Aires: FCE.

Barley, N. (1989). El antropólogo inocente. Barcelona: Anagrama.

Bawin-Legros, B. (2004). Intimacy and the New Sentimental Order, Current Sociology, 52 (2): 241250.

Beck, U. y Beck-Gernsheim, E. (1998): El normal caos del amor. Barcelona: Roure.

Caïs, J.; Folguera, L. y Formoso, C. (2014). Investigación Cualitativa Longitudinal. Cuadernos Metodológicos, 52. Madrid: CIS. 
Charsley, K. (Ed.) (2012) Transnational marriage. New Perspectives from Europe and Beyond. New York: Routledge.

Comaroff, J. y Comaroff, J. (1992). La etnografía y la imaginación histórica. Boulder: Westview Press.

Conklin, H. C. (1988). Etnografía. En J. R. Llobera, La Antropología como ciencia : 153-163. Barcelona : Anagrama.

Constable, N. (2003). Romance on a global stage. Berkeley: University of California Press.

Djurdjevic, M. y Roca, J. (2016). Mixed couples and critical cosmopolitanism: Experiences of crossborder love, Journal of Intercultural Studies, 37 (4): 390-405.

Featherstone, M. (Ed.) (1990). Global culture. Nationalism, globalization and modernity. Londres: Sage.

Ferguson, J. y Gupta, A. (1997). The field as site, method, and location in anthropology. En: A. Gupta y J. Ferguson (Eds.), Anthropological locations. Boundaries and grounds of a field science: 1-46. Berkeley, University of California Press.

Ferrándiz, F. (2011). Etnografías contemporáneas. Barcelona: Anthropos.

Foote Whyte, W. (1971 [1943]). La sociedad de las esquinas. México: Diana.

Frankenberg, R. (1980). Estudios sobre comunidades británicas. Problemas de síntesis. En: M. Banton (Comp.). Antropología social de las sociedades complejas: 134-162. Madrid: Alianza.

García Jorba, J. M. (2000). Diarios de campo. Madrid: Centro de Investigaciones Sociológicas.

Geertz, C. (1989). La interpretación de las culturas. Barcelona: Gedisa.

Geertz, C. (1989a). El antropólogo como autor. Barcelona: Paidós.

Giddens, A. (2000). La transformación de la intimidad. Madrid: Cátedra.

Hammersley, M. y Atkinson: (1994). Etnografía. Barcelona: Paidós.

Hannerz, U. (1998). Conexiones transnacionales. Madrid: Cátedra.

Hine, C. (2004). Etnografía virtual. Barcelona: UOC.

Illouz, E. (1998). The lost innocence of love. Romance as a postmodern condition. En M. Featherstone (Ed.), Love \& Eroticism: 161-186. London: Sage.

Jacobson, D. (1996). Contexts and cues in Cyberspace: The Pragmatics of naming in text-based virtual realities. Journal of Anthropological Research, 52: 461-479.

Jacobson, D. (1999) Impression formation in Cybersapce: Online expectations and offline experiences in text-based virtual communities. Journal of Computer-Mediated Communication, (5) 1. Recuperado de http://www.ascusc.org/jcmc/vol5/issue1/jacobson. html\#VirtualCommunities

King, R. (2002) Towards a New Map of European Migration”. International Journal of Population Geography, 8: 89-106.

Latour, B. (1993) Nunca hemos sido modernos. Ensayo de antropología simétrica. Madrid : Debate

Malinowski, B. (1989). Diario de campo en Melanesia. Madrid: Júcar.

Marcus, G. (1995). Ethnography in/of the world system: The Emergence of multi-sited ethnography. Annual Review of Anthropology, 24: 95-117.

Marcus, G. y Fischer, M. (1986). Anthropology as cultural critique: An Experimental moment in the Human Sciences. Chicago: University of Chicago Press.

Mayans, J. (2002). Género chat o cómo la etnografía puso un pie en el ciberespacio. Barcelona: Gedisa.

Miller, D. y Slater, D. (2000). The Internet: An Ethnographic approach. New York: Berg.

Navarro Alacalá-Zamora: (1984). Los estudios de comunidad: posibilidades y limitaciones metodológicas. En S. Rodríguez Becerra (Comp.) Antropología Cultural de Andalucía: 61-90. Sevilla: Departamento de Antropología y Floklore, Instituto de Cultura Andaluza.

Perret, G. (2011). Territorialidad y práctica antropológica: desafíos epistemológicos de una antropología multisituada/multilocal, Kula, 4: 52-60.

Pujadas, J. J.; Comas d'Argemir, D. y Roca, J. (2010). Etnografía. Barcelona, UOC.

Rabinow: (1992). Reflexiones sobre un trabajo de campo en Marruecos, Madrid: Júcar.

Rheingold, H. (1996). La comunidad virtual: una sociedad sin fronteras. Barcelona: Gedisa.

Rivers, W. H. R. (1975). El método genealógico de investigación antropológica. En J. R. Llobera (Comp.), La antropología como ciencia: 85-95. Barcelona: Anagrama.

Robertson, R. (1992). Globalization, social theory and global culture. Londres: Sage. 
Roca Girona, J. (2016). El color del deseo. Hombres españoles y mujeres eslavas heterosexuales en el supermercado global de las relaciones sexo-amorosas, Sociología Histórica, 6: 389-426.

Roca Girona, J. (2016a). De Sur a Norte, de Norte a Sur: el balance laboral de mujeres cualificadas migrantes por amor, Revista Andaluza de Antropología, 11: 92-120.

Roca Girona, J. (2014). [Per] siguiendo informantes en terrenos movedizos. Una reflexión a partir de una experiència etnogràfica con migrantes por amor. En C. Oehmichen (Ed.) La etnografía y el trabajo de campo en las ciencias sociales: 305-321. México: Universidad Nacional Autónoma de México.

Roca Girona, J. (2011). Amores glocales, noviazgos transnacionales. La búsqueda virtual de pareja mixta por parte de hombres españoles, Revista de Antropología Social, 20: 263-292.

Roca Girona, J. (2011a). [Re]buscando el amor: Motivos y razones de las uniones mixtas de hombres españoles con mujeres extranjeras, Revista de Dialectología y Tradiciones Populares, LXVI: 487-514.

Roca Girona, J. (2010). Los estudios de comunidad. En J. J. Pujadas; D. Comas d'Argemir y J. Roca, Etnografía: 214-226. Barcelona: UOC.

Roca Girona, J. (2010a). Las etnografías de orientación temática. En J. J. Pujadas; D. Comas d'Argemir y J. Roca, Etnografía: 255-270. Barcelona: UOC.

Roca Girona, J. (2009). Migraciones amorosas, migraciones (re)negadas, Migraciones, 25: 89-124.

Roca Girona, J. (2009a). 'Quien lejos se va a casar..' Migraciones (re)negades. Alteridades, 19: 133155.

Roca Girona, J. (2007). Migrantes por amor. La búsqueda y formación de parejas transnacionales, AIBR, 2: 430 - 458.

Roca, J. y Allué, M. (2016). Amores lejanos. Historias de parejas transnacionales. Barcelona: Bellaterra.

Roca, J., Anzil, V. y Martínez, L. (2015). Intimacies turned on and off. Spanish men in search of a foreign partner beyond the screen. En B. Enguix and J. Roca (Eds.) Rethinking Romantic Love: Discussions, Imaginaries, and Practices: 61-100. Cambridge Scholars Publishing.

Roca, J. y Urmeneta, A. (2013) Bi-national weddings in Spain: A Recent and increasingly frequent phenomenon in the context of the globalization of the marriage market", Procedia - Social and Behavioral Sciences, 82: 567-573.

Roca Girona, J. (Dir.), Bodoque, Y., Djurdjevic, M., Martínez, L., Soronellas, M. (2013) Migrantes por amor. La búsqueda de pareja en el escenario transnacional. Valencia: Germania.

Shumway, D. (2003). Modern love: Romance, intimacy and the marriage crisis. New York: N. Y. Univ. Press.

Terradas Saborit, I. (1991). La historia de las estructuras y la historia de la vida. Reflexiones sobre las formas de relacionar la historia local y la historia general. En J. Prat; U. Martínez; J. Contreras e I. Moreno (Eds.) Antropología de los Pueblos de España: 159-176. Madrid: Taurus.

Williams, L. (2010). Global marriage. Cross-border marriage migration in global context, London: Palgrave Macmilan.

Willis: (2000). The Ethnographic imagination. Cambridge: Polity Press.

Zelizer, V. A. (2005). The Purchase of intimacy. Princeton: Princeton University Press.

\section{3}

